\title{
The "transformer" model of ROS management for PD and cancer: Network diversification as the source of precision in blue-print modelling
}

\author{
A. Kolodkin ${ }^{1,2,3,4,5 *}$, A.M. Colangelo ${ }^{6}$, L. Alberghina ${ }^{6}$, R.P. Sharma ${ }^{7}$, \\ V. Kumar ${ }^{7}$, D. Jennen ${ }^{8}$, J.J. Briede ${ }^{8}$, H.V. Westerhoff ${ }^{1,4,5,9}$ \\ ${ }^{1}$ Infrastructure for Systems Biology Europe (ISBE) \\ ${ }^{2}$ Luxembourg Centre for Systems Biomedicine, University of Luxembourg, Luxembourg \\ ${ }^{3}$ Federal Centre Institute of Cytology and Genetics of Siberian Division of the Russian Academy \\ of Sciences, Russia \\ ${ }^{4}$ Molecular Cell Physiology, VU University Amsterdam, The Netherlands \\ ${ }^{5}$ Synthetic Systems Biology, SILS, University of Amsterdam, The Netherlands \\ ${ }^{6}$ SysBio Centre of Systems Biology, University of Milano-Bicocca, Italy \\ ${ }^{7}$ Center of Environmental Food and Toxicological Technology (TecnATox), Departament d'Enginyeria \\ Química, Universitat Rovira i Virgili, Spain \\ ${ }^{8}$ Department of Toxicogenomics, Maastricht University, The Netherlands \\ ${ }^{9}$ Manchester Centre for Integrative Systems Biology, UK \\ *e-mail: a.n.kolodkin@vu.nl
}

Key words: systems biology, dynamic modelling, blueprint modelling, cancer, Parkinson's disease

Biomolecular and functional similarity of various cell types allows constructing a universal blueprint model of the cell. Most processes and qualitative descriptions of biomolecular interactions should be similar. They would differ in kinetic parameter values and expression levels. Re-parameterization should allow switching the model from one cell type, e.g. hepatocyte to another, e.g. dopaminergic neuron. This resembles transformers - children toys that can change shape from humanoid, to vehicle, to weapon, with just a few simple turns.

We have built a blueprint model of ROS management in several instantiations, e. g. hepatocyte and neuron-like cells. Then we used two independently obtained data sets showing the response of those cells to oxidative stress. Fitting the model to one data set, should somewhat enhance the confidence to the model describing another data set. This is then an example of how blueprint modelling of the cell may become more and more precise by taking into account various instantiations and data sets.

This blueprint concept goes hand-in hand with implications for building a universal model for different diseases. Striking examples are Parkinson's disease and cancer, which look like opposing diseases: in cancer the cell lives, in PD the cell dies. Our ROS model explains both cases by demonstrating how an overexpression of just one protein (DJ1), relevant for some cancers, makes cell "immortal" under oxidative stress, while downregulation of the same protein, which is relevant for some cases of PD, accelerates cell death under oxidative stress. 